Introduction Malignant Pleural Mesothelioma (MPM) is an aggressive cancer caused by occupational, environmental and indirect exposure to asbestos, material that some countries have already prohibited. Survival is less than 1 year. Diagnosis is currently a challenge and the search for early, single or combined diagnostic biomarkers continues to be performed on non-invasive samples. MicroRNAs are circulating released molecules whose expression is altered in some types of cancer, making them good diagnostic candidates in the MMP.

Methods A descriptive study was carried out in plasma of 6 cases of MPM and 6 controls without exposure to asbestos matched by age and sex. The samples come from a previous case-control study conducted in Mexico from 2011 to 2016. We evaluated 384 plasma microRNAs by means of RT-qPCR using the platform and QIAgen. The analysis of results was performed with the GeneGlobe program.

Results hsa-miR-1587, hsa-miR-19b-1-5 p, hsa-miR-93-3 p, hsa-miR-21-3 p, hsa-let-7i-3p, hsa-miR-194-5 p, hsa-miR-1280, hsa-miR-18a-3p, hsa-miR-133a-3p, hsa-miR-4286, hsa-miR2467-3 p, had altered expression in cases and controls

Discussion While some microRNAs found in this study have been associated with kidney disease, hypertension, endometriosis, liver cancer, diabetes, colorectal cancer, gastric and esophageal cancer; The microRNA hsa-let-7i-3p associated with lung cancer are specially interest, miR-194-5 $\mathrm{p}$ deregulated in tumorogenesis and miR-1280 modulates cell growth, so the next step will be to validate them in a new collection of samples and perform the network of interaction in the metabolic pathways.

\section{SEGMENTARY DEMYELINATING POLYNEUROPATHY AS A SEQUEL BY ARSINE GAS INTOXICATION. CASE REPORT}

${ }^{1} \mathrm{~A}$ Bajeux*, 'GA Real, ${ }^{1} \mathrm{MG}$ Valdés, ${ }^{2} \mathrm{~J}$ Figueroa. ${ }^{1}$ UIDAC, Research Unit, Teaching and Clinical Support in Occupational Health, IMSS, Guadalajara, Mexico; ${ }^{2}$ DIST, Division of Occupational Health, IMSS, Guadalajara, Mexico

\subsection{6/oemed-2018-ICOHabstracts. 1162}

Introduction Arsine gas intoxication generates enzymatic changes on glutathione in erythrocytes, leading to massive hemolysis, which manifests as altered consciousness, hematuria, jaundice, and renal failure. Its importance lies in the early diagnosis for the limitation of its sequelae.

Methods 26-year-old worker, operator in the area of polymetals, who in June 2016, after adding zinc in a mixing tank, starts with ocular ardour, upper respiratory tract irritation, nausea and vomiting. Initially treated as gastroenteritis, whithout response to treatment. Evaluated by internal medicine diagnosing hemolytic uremic syndrome and liver failure requiring hospitalisation. Presenting later short-term memory loss, vertigo, pain and weakness in pelvic limbs. Evaluated by neurology granting symptomatic management and requesting electromyography of pelvic limbs, which reported segmental demyelinating polyneuropathy. Valorated by the occupational health division, where a specific study was carried out observing the inconsistent use of personal protective equipment, as well as the inadequate manipulation of the zinc as causative factors of intoxication, determining neuropathy as a sequel.
Result Laboratory: arsenic: in urine $715.0 \mu \mathrm{g} / \mathrm{L}$, in blood $95 \mu \mathrm{g} / \mathrm{L} ; \mathrm{Hb} 7.1 \mathrm{~g} / \mathrm{dl}$, Hct $28 \%$, creatinine $3.2 \mathrm{mg} / \mathrm{dl}$, urea $149 \mathrm{mg} / \mathrm{dl}$, urinalysis: nitrites + , proteins $500 \mathrm{mg} / \mathrm{dl}$, haematuria. Physical examination: paraesthesia and dysaesthesias of pelvic limbs, muscle strength $3 / 5$ bilateral, electromyography of pelvic limbs: motor polyneuropathy of the type of segmental demyelination.

Discussion Exposure to arsine gas without adequate personal protective equipment can lead to severe intoxication, develop of sequelae and even death. In the present case the process by which arsine gas is produced in the workplace is compatible with the symptoms and sequelae presented by the worker, demonstrating the cause-effect work-injury relationship.

\section{DEFINING THE CONTRIBUTION OF DIETARY SOURCES OF FURFURAL METABOLITES IN URINE: IMPLICATIONS FOR BIOMONITORING AND RISK ASSESSMENT}

${ }^{1} \mathrm{~A}$ Bonari* ${ }^{*}{ }^{2} \mathrm{~S}$ Dugheri, ${ }^{1} \mathrm{I}$ Pompilio, ${ }^{1} \mathrm{C}$ Bossi, ${ }^{1} \mathrm{~N}$ Mucci, ${ }^{1} \mathrm{M}$ Montalti, ${ }^{1} \mathrm{G}$ Arcangeli. ${ }^{1}$ Department of Experimental and Clinical Medicine, University of Florence, Firenze, Italy; '2Laboratorio di Igiene e Tossicologia Industriale, Azienda Ospedaliero-Universitaria Careggi, Firenze, Italy

\subsection{6/oemed-2018-ICOHabstracts.1163}

Introduction Furfural is an organic compound derived from a variety of agricultural products, and it is mainly used as petrochemical solvent and in rubber industries. In a wide range of food heating processes furfural may rise from 5-hydroxymethylfurfural (5-HMF), a product of dehydration of sugars and Maillard reactions. Our aim is the development of analytical approach to determine the common urinary metabolism of furfural and 5-HMF, by quantification of 2- and 3- furoic acids (FA), 5-HMF itself and its specific metabolite, 5-hydroxymethyl-2-furoic acid (HMFA).

Methods A group of healthy control subjects received an oral dose of 5 -HMF in plum juice $(780 \mathrm{mg})$ and its relative metabolites the investigated in urine within 24 hours. Analysis were carried out by solid-phase microextraction and gas chromatography/mass spectrometry. FAs were determined in head space after the conversion into their methyl esters derivatives by a reaction with trimethyloxonium tetrafluoroborate. HMFA tertbutyldimethylsilyl derivative was detected by direct immersion using $\quad \mathrm{N}$-tert-butyldimethylsilyl- $\mathrm{N}$-methyltrifluoroacetamide while 5-HMF quantification was perfomed by on sample derivatization with O-(2,3,4,5,6-pentafluorobenzyl) hydroxylamine. Results Data from 22 subjects were analysed. Mean daily concentrations of 2-FA, 5-HMF were quantified $46.1 \pm 15.1 \mathrm{mg} /$ 24 hour, $7.53 \pm 2.11 \mathrm{mg} / 24$ hour. HMFA and 3-FA were not detected in all urine samples. 2-FA and 5-HMF concentration resulted particularly higher within 6 hours after the plum juice administration (mean of $37.1 \mathrm{mg} / \mathrm{L}$ and $5.3 \mathrm{mg} / \mathrm{L}$ respectively). Conclusion Total urinary FAs may not discriminate a food intake or environmental exposures. Significant increases of 2and 3-FA concentrations from professional exposure are identified, while high values of 2-FA alone are found after the intake of food with elevated quantity of HMF. Therefore total FA as Biological Exposure Index by the American Conference of Governmental Industrial Hygienists, could lead to unsuitable assessment of the level of furfural in the workplace. 